\title{
Experimental and Theoretical Evaluation of an Ultrafast Multihop Packet-Switched Optical TDM Network Testbed
}

\author{
Paul Toliver, Ben Y. Yu, Robert J. Runser, Kung-Li Deng, \\ Deyu Zhou, Tian Chang, Koo I. Kang, Ivan Glesk, \\ and Paul R. Prucnal
}

\author{
Department of Electrical Engineering \\ Princeton University \\ Princeton, NJ 08544 \\ Tel: 609-258-2041; Fax: 609-258-2158 \\ Email: ptoliver@ee.princeton.edu
}

Abstract-We discuss some of the physical layer performance issues regarding an experimental multihop transparent optical packet-switched testbed developed recently at Princeton University. Experimental data as well as computer simulation results are presented. 


\title{
Experimental and Theoretical Evaluation of an Ultrafast Multihop \\ Packet-Switched Optical TDM Network Testbed
}

\author{
Paul Toliver, Ben Y. Yu, Robert J. Runser, Kung-Li Deng, \\ Deyu Zhou, Tian Chang, Koo I. Kang, Ivan Glesk, and Paul R. Prucnal \\ Department of Electrical Engineering, Princeton University \\ Princeton, NJ 08544
}

Tel: 609-258-2041; Fax: 609-258-2158

Email: ptoliver@ee.princeton.edu

Recent advances and innovations in ultrafast optical TDM technologies will be able to provide for the basic building blocks required in the next generation of high-speed processor interconnects and computer LANs. One network that has integrated many OTDM technologies such as $100 \mathrm{~Gb} / \mathrm{s}$ packet compression and ultrafast demultiplexing has recently been demonstrated at Princeton University ${ }^{1}$. The network logical and physical topology is based upon the ShuffleNet architecture ${ }^{2,3}$. This paper briefly presents the experimental results of this demonstration and addresses the physical layer performance issues including scalability, reliability, and channel constraints of such networks.

In the system demonstrations, packet data was first encoded onto a $100 \mathrm{MHz} 1 \mathrm{ps}$ pulse stream at $1313 \mathrm{~nm}$ and then compressed to a pulse spacing of $10 \mathrm{ps}$ using a 4-stage feed-forward delay line structure ${ }^{4}$. A novel packet header scheme that takes advantage of the regular structure of the ShuffleNet architecture was implemented to allow the network to be easily scalable ${ }^{5}$. The packet header demultiplexing subsystem was constructed using a parallel array of three Terahertz Optical Asymmetric Demultiplexers (TOAD's) ${ }^{6}$, and a routing controller routed the incoming packets appropriately through the node's $4 \times 4$ routing switch. Since only one physical node of this network was constructed and tested, the entire ShuffleNet was synthesized by using a feedback fiber and allowing the node to dynamically reconfigure its current node address as the 
packet traversed the network. An example routing path through the ShuffleNet is shown in the inset of Fig. 1 as well as the output of the experimental node $4 \times 4$ routing switch. In this demonstration, a new $100 \mathrm{~Gb} / \mathrm{s}$ packet was regenerated at each virtual node in order to demonstrate functional operation of the demultiplexing subsystem and routing controller. In Fig. 2, the evolution of the packet when the packet is simply looped back to the node input is illustrated. In this setup, one semiconductor optical amplifier was used to compensate as much round-trip loss as possible, but the gain was not adequate to exactly compensate for the loss, which explains the exponential decay of the packet.

The physical layer effects that impact the system performance include the dispersive and nonlinear effects of the fiber links, optical amplifier noise accumulation, adjacent channel crosstalk noise accumulation due to imperfect extinction ratio of lithium niobate routing switches (approximately $22 \mathrm{~dB}$ for commercially available devices), and gain dynamics of the SOA used in the TOAD. A computer simulation program was developed to model the physical layer of the network. In Fig. 3, an eye diagram after five hops (inset) and the SNR penalty as a function of the number of hops is illustrated. The simulation takes into account all of the effects described above and assumes the round trip loss can be compensated. The primary source of the SNR degradation is due to crosstalk and can be improved significantly with the development of higher extinction ratio space switches or use of alternative switch topologies. 


\section{REFERENCES}

1. B. Y. Yu, R. Runser, P. Toliver, K. L. Deng, D. Zhou, I. Glesk, and P. R. Prucnal, “A network demonstration of $100 \mathrm{Gbit/s}$ optical packet switching with self-routing", Electron. Lett. 33, 1401 (1997)

2. M. G. Hluchyj, and M. J. Karol, "Shufflenet: An application of generalized perfect shuffles to multihop lightwave networks", J. of Lightwave Technol. 9, 1386 (1991)

3. A. S. Acampora, "The Scalable Lightwave Network", IEEE Comm. Mag. 32, 36 (1994)

4. K. L. Deng, K. I. Kang, I. Glesk, and P. R. Prucnal, "A 1024-channel fast tunable delay line for ultrafast all-optical TDM networks”, IEEE Photon. Technol. Lett. 9, 1496 (1997)

5. S. Seo, B. Y. Yu, and P. R. Prucnal, "Bit-level packet-switching all-optical multihop shuffle networks with deflection routing", Appl. Opt. 36, 3142 (1997)

6. J. P. Sokoloff, P. R. Prucnal, I. Glesk, and M. Kane, "A terahertz optical asymmetric demultiplexer (TOAD)", IEEE Photon. Technol. Lett. 5, 787 (1992) 


\section{FIGURE CAPTIONS}

- Figure 1: Routing path (inset) and $4 \times 4$ routing switch outputs for the experimental demonstration

- Figure 2: Experimental setup (inset) and evolution of the packet through the ShuffleNet using the fiber loopback and semiconductor optical amplifier

- Figure 3: Eye diagram after the fifth hop (inset) and SNR degradation as a function of the number of hops 


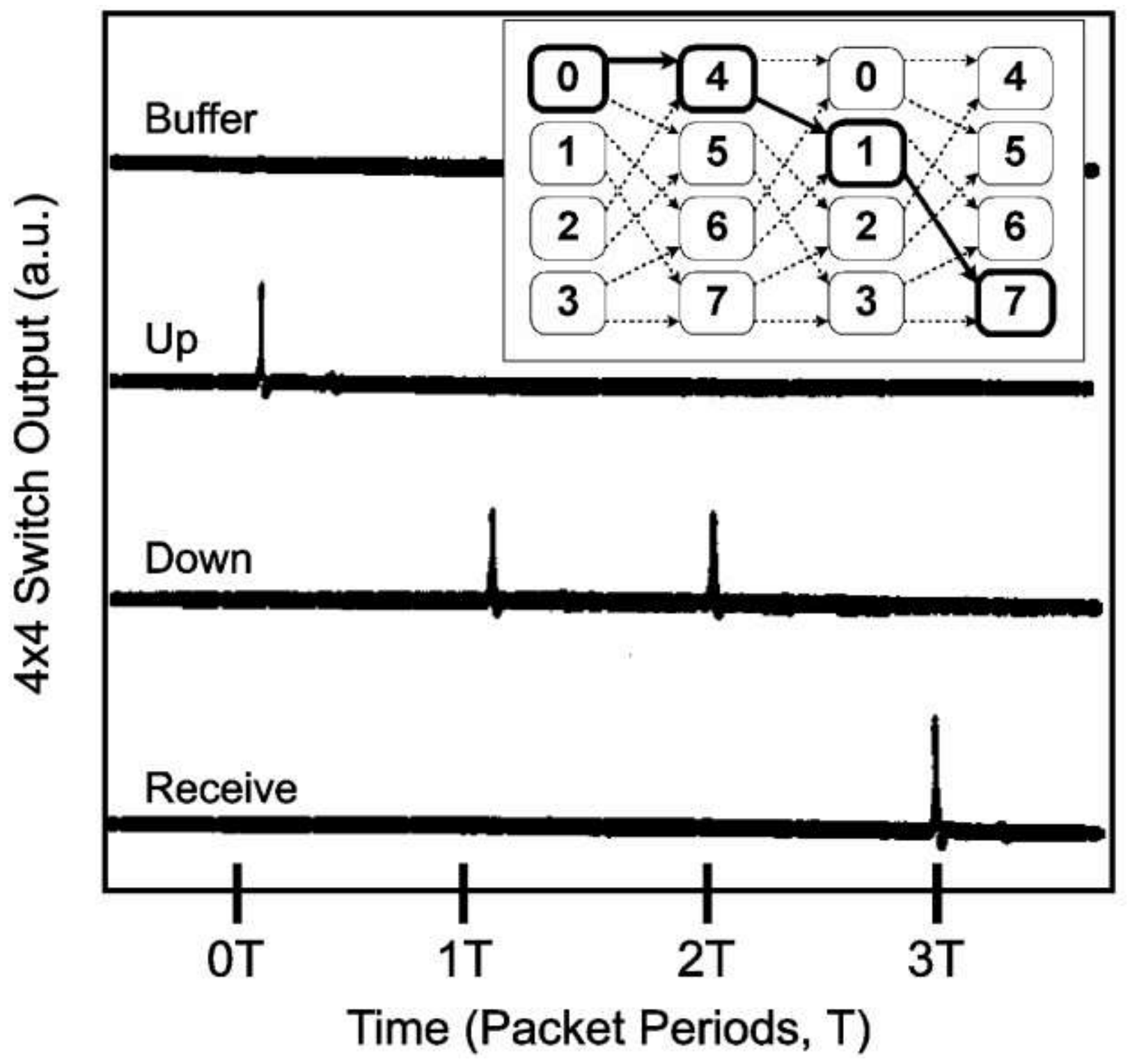

Fig. 1 


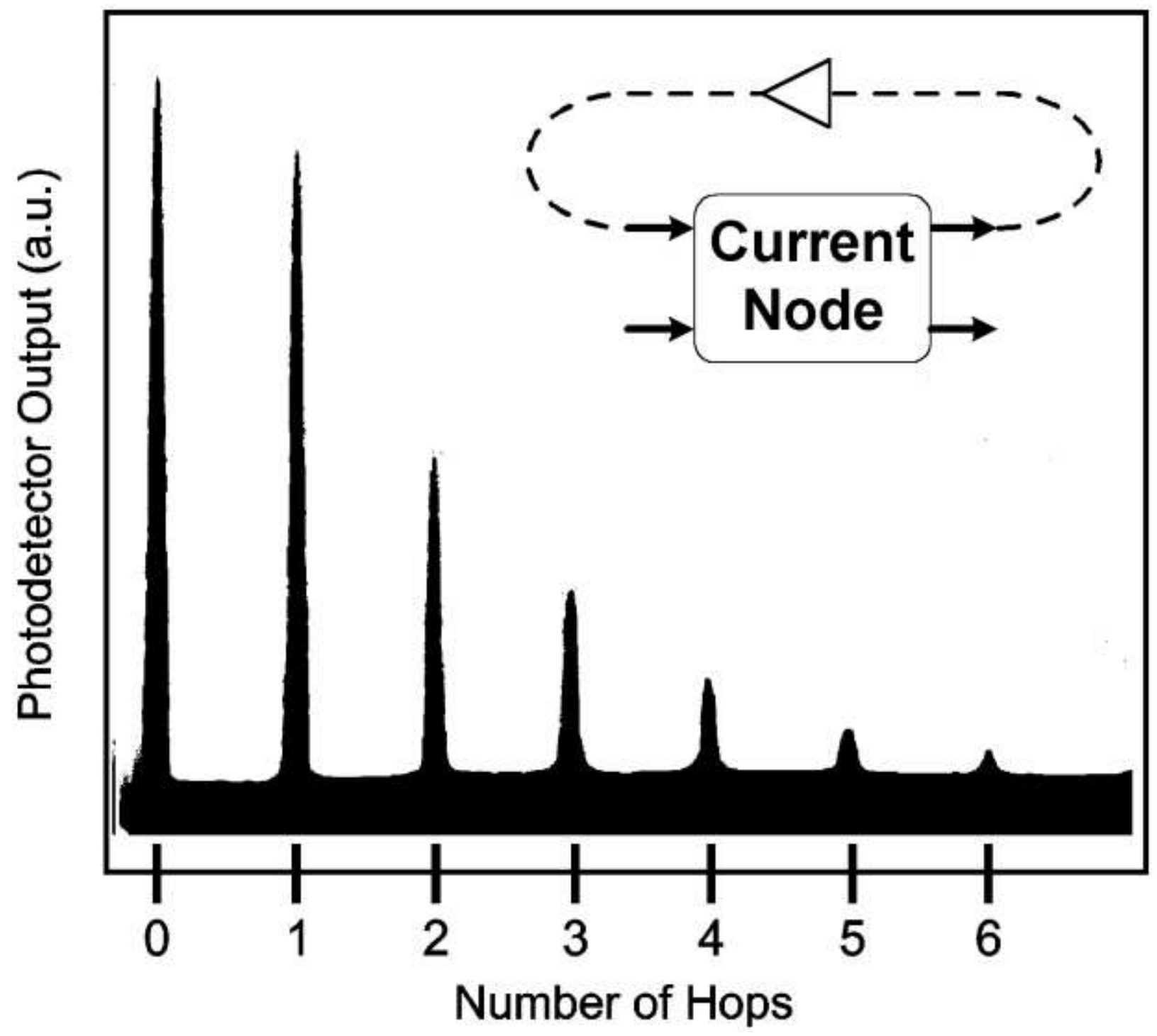

Fig. 2 


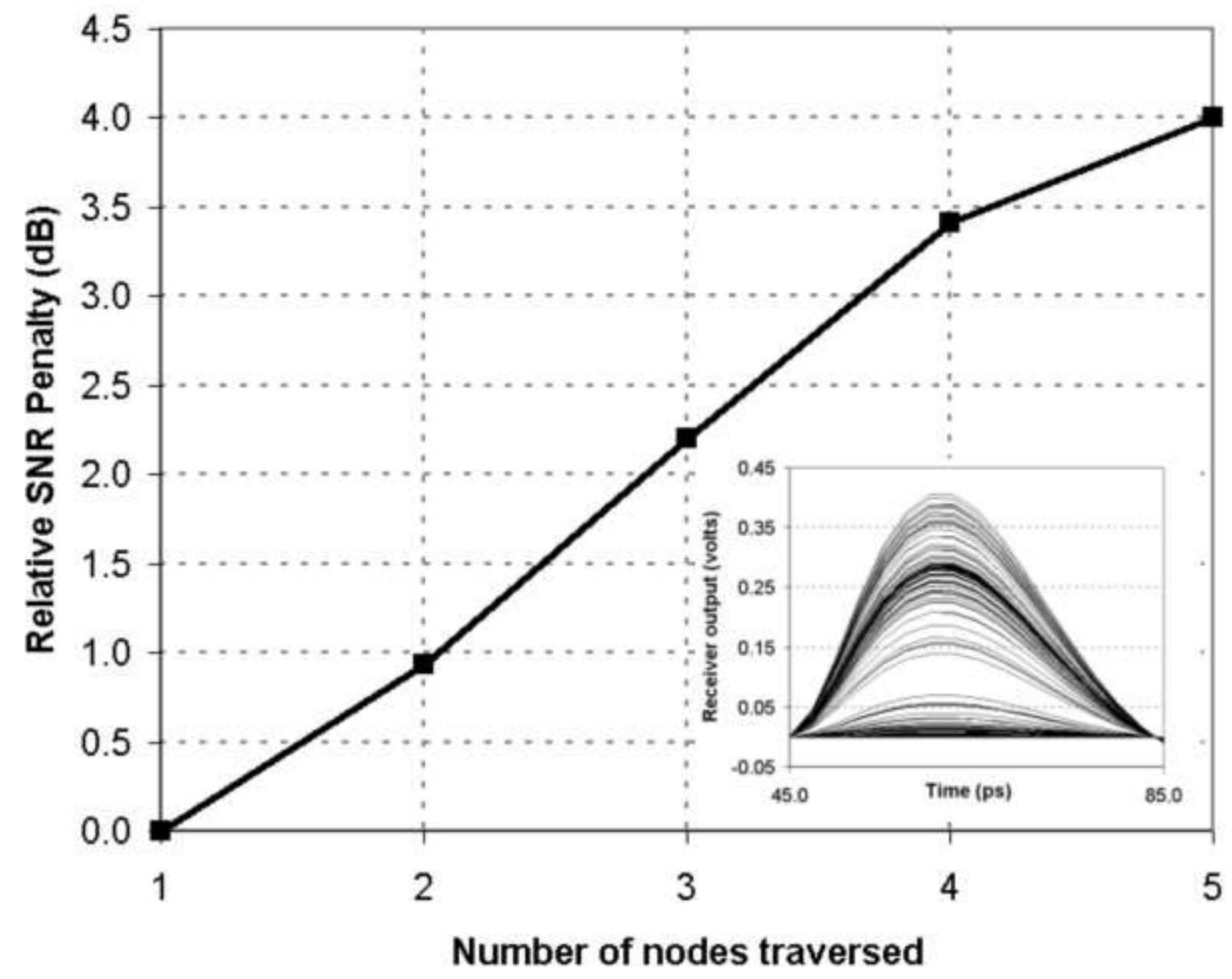

Fig. 3 